\title{
Wortverbindungen - mehr oder weniger fest
}

Herausgegeben von

Kathrin Steyer

Walter de Gruyter • Berlin • New York 


\section{Redaktion: Franz Josef Berens}

(@) Gedruckt auf säurefreiem Papier, das die US-ANSI-Norm über Haltbarkeit erfüllt.

\section{ISBN 3-11-0177956-3}

\section{Bibliografische Information Der Deutschen Bibliothek}

Die Deutsche Bibliothek verzeichnet diese Publikation in der Deutschen Nationalbibliografie; detaillierte bibliografische Daten sind im Internet über http://dnb.ddb.de abrufbar.

\section{(C) Copyright 2004 by Walter de Gruyter GmbH \& Co. KG, D-10785 Berlin}

Dieses Werk einschließlich aller seiner Teile ist urheberrechtlich geschützt. Jede Verwertung außerhalb der engen Grenzen des Urheberrechtsgesetzes ist ohne Zustimmung des Verlages unzulässig und strafbar. Das gilt insbesondere für Vervielfältigungen, Übersetzungen, Mikroverfilmungen und die Einspeicherung und Verarbeitung in elektronischen Systemen.

Printed in Germany

Satz: Greiner \& Reichel, Köln

Einbandgestaltung: Christopher Schneider, Berlin 


\title{
VILMos ÁGEL
}

\section{Phraseologismus als (valenz)syntaktischer Normalfall}

\begin{abstract}
Mehr oder weniger feste Wortverbindungen stellen keine Sonder-, sondern vielmehr Normalfälle sprachlicher Zeichenbildung dar. In krassem Gegensatz zum sprachlichen Normalstatus steht allerdings ihr linguistischer Reststatus in der traditionellen Theoriebildung. Wenn man zum Minimalkriterium deskriptiver Adäquatheit macht, gewöhnliche sprachliche Phänomene mit gewöhnlichen Mitteln einer linguistischen Theorie zu beschreiben, so ergibt sich aus der Statusspannung eine anspruchsvolle Aufgabe für künftige Theorien. Vorliegender Aufsatz stellt einen ersten Versuch dar, einen valenztheoretischen Beitrag zum phraseologischen Sprachnormalfall zu leisten.
\end{abstract}

\section{Valenz in der Phraseologieforschung}

Die valenzbezogenen Themen der PH-Forschung sind einerseits herkömmliche Themen der Valenztheorie wie die E/A- und die o/f-Unterscheidung.' Andererseits gibt es aber auch speziell phraseologisch ,erzeugte' valenz(theorie)bezogene Themen (s. auch den Forschungsüberblick in Wotjak 1992, S. $47 \mathrm{ff}.):^{2}$

(1) Interne/innere vs. externe/äußere Valenz (vgl. Abschnitt 4);

(2) Transparenz/Opakheit und Valenz (Hessky 1988, S. 147 f.);

(3) Lesarttypen und Valenz (Burger 1989; Burger 1998, S. 63 ff.; Wotjak 1992, S. 35 f.);

(4) Valenz und Variabilität (vgl. Abschnitt 4.1);

(5) Strukturmodelle von Idiomen (Fix 1974/76 und 1979);

(6) Phraseoschablonen (Fleischer 1997, S. $130 \mathrm{ff}$.).

Teils im Zusammenhang mit den genannten Themen, teils unabhängig von ihnen können aus valenztheoretischer Sicht folgende Fragen formuliert werden. Die Reihenfolge der Fragen stellt keine Rangfolge dar:

\footnotetext{
Abkürzungen im fließenden Text: $E=$ Ergänzung; $A=$ Angabe; $o=$ obligatorisch; $f=$ fakultativ; VT $=$ Valenzträger; AP $=$ Aktantenpotenzial; NVG $=$ Nominalisierungsverbgefüge; FVG = Funktionsverbgefüge; $\mathrm{PH}=$ Phraseologismus, ... men; PH-Forschung/-Forscher $=$ Phraseologieforschung/-forscherInnen. Zu den Abkürzungen für einzelne Valenzrelationen vgl. Anm. 29.

${ }^{2}$ Nicht speziell valenztheoretische, sondern allgemein syntaktische und somit auch die Valenz betreffende Themen sind die grammatischen Verhaltenseigenschaften von PH wie etwa Passivierung (Iker 1996; Dobrovol'skij 2000).
} 
(1) Warum wird eine ganze Reihe valenztheoretisch relevanter polylexikalischer Einheiten, die unter bestimmten theoretischen Bedingungen den Minimalkriterien von Phraseologizität (Polylexikalität und Fixiertheit) entsprechen, aus der PH-Forschung ausgeklammert? ${ }^{3}$

(2) Warum werden die strukturellen Unterschiede zwischen nominativen (= akkusativsprachlichen) und absolutiven (= ergativsprachlichen) $\mathrm{PH}$ (jmdm. raucht der Kopf; mir brummt/schwirrt der Kopf; jmdm. geht ein Licht auf) nicht berücksichtigt?

(3) Wie ist die Grenze zwischen Idiom und dessen Umgebung valenztheoretisch zu erfassen?

(4) Wie ist Variation (Burger 1998, S. $25 \mathrm{ff}$.) valenzträgertheoretisch zu erfassen?

(5) Wie sind kreativ-wortspielerische Modifikationen (Wotjak 1992, S. $161 \mathrm{ff}$.) und vor allem wie ist die systematische Modifikation (Keil 1997, S. 72) valenzträgertheoretisch zu erfassen?

(6) Welche valenztheoretischen Implikationen und Konsequenzen hat die Unterscheidung zwischen interner und externer Valenz?

\section{Phraseologie in der Valenztheorie}

Die phraseologische Thematik in der Valenztheorie steht überwiegend unter dem Zeichen eines Rest- und Sonderfalls. Aus dieser schiefen Perspektivierung folgt, dass man sich in der Valenztheorie weniger um der Phraseologie willen mit PH beschäftigt hat, sondern eher mit dem Ziel, theorieinterne Probleme zu lösen. Denn Polylexikalität - ob Verbalkomplexe, Partikelverben, Kopulakonstruktionen, NVG, FVG oder Idiome - stellt seit eh und je ein kritisches Problem für Valenztheorien dar (s. Ágel 2000, S. 138 ff.). Entsprechend steht in valenztheoretischen Arbeiten zur Phraseologie

(1) die ,Einpassbarkeit“ verbaler Phraseologismen (von Idiomen und NVG) in das gegebene Valenzmodell

im Zentrum (kritisch s. Van Pottelberge 2001, S. $37 \mathrm{ff}$.). Alles andere lässt sich als theoretischer Ausfluss der ,Einpassbarkeitsthematik' begreifen. Vor allem geht es dabei um folgende Themen:

(2) Valenzstruktur und Bedeutungsstruktur (Wotjak 1992);

(3) die Relation zwischen semantischer und syntaktischer Valenz (ebd.);

${ }^{3}$ Ich denke an relationale Sprachzeichen mit ihren präpositionalen Rekta (nachdenken über, bekannt für usw.), an sich-Verben mit medialem sich (s. Ágel 1997, S. 182 f.), an Verben mit es impersonale und an idiomatisch geprägte labile Zweiwortkonstruktionen (ist zerbrochen; ist geschmolzen usw., s. Ágel 2003). Lösungsvorschläge würden eine Auseinandersetzung mit der Problematik der Polylexikalität voraussetzen (zu einem Versuch vgl. Ágel 2003a), die wiederum eine allgemein-sprachwissenschaftliche Auseinandersetzung mit dem (zentralen) phraseologischen Kriterium der Fixiertheit voraussetzt (Schemann 1987). 
(4) ,besondere' Leerstellen (Korhonen 1988, S. 106 f. und 1995, S. 82; Hyvärinen 2003, S. 42 f.) wie z. B. die Genitivstellen in den PH jmds. Thron wackelt, in jmds. Fußstapfen treten oder die Präpositionalstelle in eine Antenne für etw. haben (Hessky 1988, S. 145).

Aus der Sicht der PH-Forschung könnten aber auch Antworten z. B. auf die folgenden Fragen erwartet werden:

(1) Warum befasst sich die Valenztheorie nur mit einigen wenigen Klassen von Phraseologismen? Wäre eine Gegenstandsextension etwa in Richtung auf pragmatische $\mathrm{PH}$ angebracht? ${ }^{4}$

(2) Sollen PH weiterhin als Rest- und Sonderfälle des VT behandelt werden?

(3) Wie geht man mit dem Problem der semantischen Autonomie von Idiomkonstituenten (etwa im Sinne von Dobrovol'skij 1995, S. 27 ff.) um?

(4) Kann die Valenztheorie die systematische Modifikation nur als Systemoder Normabweichung modellieren?

(5) Kann die Valenztheorie dazu beitragen, $\mathrm{PH}$ nicht mehr grundsätzlich als idiosynkratisch anzusehen?

\section{Neuorientierungen}

Es sind sowohl im Umkreis der PH-Forschung als auch in der Valenztheorie Ansätze zu einer theoretischen Neuorientierung zu beobachten.

In der einen massiven extensionalen und intensionalen Wandel erfahrenden PH-Forschung geht es im Grunde um die Erkenntnis, dass sich Kreativität und Routine (Stein 1995, S. $108 \mathrm{ff}$.) nicht ausschließen, sondern sich im Gegenteil gegenseitig im Sinne eines ewigen Zirkels bedingen: Kreativität baut auf Routine auf, und Routine wird von ,unsichtbaren Händen' entworfen, deren Kreativität sich anhand von Handlungsmaximen bündeln lässt (Keller 1990, S. $121 \mathrm{ff}$.). Diese theoretische Umperspektivierung wirft nun die folgende ,Schicksalsfrage" auf:

Ist oder bleibt die Phraseologie eine Disziplin, die den lexikologischen (bzw. den grammatischen und den pragmatischen) Sonderfall zum Objekt hat, oder soll sie zu einer Disziplin werden, die den sprachlichen Normalfall zu ihrem Gegenstand macht? Zugespitzt formuliert: PH-Forschung als Restoder als Überdisziplin?

Sollte der sprachliche Normalfall nicht das Okkasionelle, sondern das Geprägte und Vorgeprägte sein, müssten Theorien und Methoden der Linguistik darauf umgestellt werden, dass die ad-hoc-Bildungen mehr oder weniger kreative Realisierungen idiomatisch geprägter und vorgeprägter Modelle darstellen, dass also die grammatischen Regeln auf der Basis dieser Modelle funktionieren. Wenn Florian Coulmas (1985, S. 48 f.) schreibt, dass der Sprachgebrauch des native speaker durch Ausdrücke, die (a) grammatisch

${ }^{4}$ Stein (1995, S. 121 f. und S. 129 ff.) spricht im Zusammenhang mit gesprächsspezifischen Formeln von ,Leerstellen'. 
und idiomatisch und (b) ungrammatisch und idiomatisch sind, gekennzeichnet sei, meint er wohl Ähnliches. Nach Helmuth Feilke (1996, S. 225 ff.) stellen die sog. Satzbaupläne „syntaktische Ausdrucksmodelle mit primär syntaktischer Prägung" (ebd., S. 239) dar. Diese Ansicht zu teilen, würde bedeuten, „daß ein enormer Teil der deutschen Syntax im weitesten Sinne als phraseologisch einzustufen wäre“, folgert Stephan Stein (1995, S. 19, Anm. 6), der seinerseits für die Pragmatik nachweist, dass Formelhaftigkeit den sprachlichen Normalfall darstellt. Gertrud Gréciano (1992, S. 155) konnte zeigen, „wie Texte in ihrer Kohäsion und Kohärenz über Phraseme erzeugt werden", was nahe legt, dass die textkonstituierende Rolle von PH ebenfalls nicht als ein Sonderfall behandelt werden kann.

Aber auch wenn manch einem PH-Forscher die Ansichten von Coulmas oder Feilke radikal erscheinen mögen, dürfte es mittlerweile klar geworden sein, dass die Gegenstandsbestimmung keine innere Angelegenheit der Phraseologie mehr sein kann, sondern im Grunde gesamtlinguistischer Anstrengungen bedarf, wobei sie entweder einen Paradigmenwechsel in der Linguistik oder dessen konsensuelle Ablehnung voraussetzt.

Auch in der Valenztheorie äußert sich die theoretische Neuorientierung in einem extensionalen und intensionalen Wandel bzw. in einer theoretischen Umperspektivierung (s. hierzu Ágel 2000). Ich beschränke mich lediglich auf die Punkte, die für den angekündigten Versuch, einen valenztheoretischen Beitrag zum phraseologischen Sprachnormalfall zu leisten, relevant sind.

Nach meiner Auffassung beschäftigt sich die Valenztheorie mit insgesamt vier Grundfragen, weshalb sie in vier Teiltheorien - in je zwei Valenzpotenzund Valenzrealisierungstheorien - zu unterteilen ist (Ágel 2000, S. 105 ff.):

Tabelle 1: Grundfragen und Teiltheorien

Valenzpotenztheorien

(I) Valenzträger (=VT)

(II) Aktantenpotenzial (= AP)
Valenzrealisierungstheorien

(III) strukturelle

(IV) kontextuell-situative

Die traditionelle Valenztheorie konzentriert sich auf die Grundfragen II und IV und vernachlässigt die Fragen I und III (zu diesen vgl. Ágel 2000, S. $113 \mathrm{ff}$. und S. $215 \mathrm{ff}$.). ${ }^{5}$ Fraglich ist dabei, ob es richtig war, die AP-Theorie zur zentralen Theoriekomponente zu machen, schließlich könnten gerade die PH-Forscher ein Lied davon singen, wie schwer es ist, die sog. Idiomkerne von ihrer Umgebung zu trennen und sie zusammen mit ihren Varianten strukturell und semantisch zu kategorisieren. Dass die Valenztheorie nur in Ansätzen über eine VT-Theorie verfügt, rächt sich also am massivsten bei der Behandlung von PH, die man immer noch als den Sonderfall des VT an-

${ }^{5}$ Auf die Theorietypen des AP (Grundfrage II) komme ich noch in Abschnitt $4.2 \mathrm{zu}$ sprechen. 
sieht. Was aber könnte eine ,neue' Phraseologie des sprachlichen Normalfalls mit einer VT-Theorie des phraseologischen Sonderfalls anfangen?

Während die Notwendigkeit, VT-Theorie und PH-Forschung zu verbinden, auf der Hand liegt, gibt es $\mathrm{m}$. W. noch überhaupt keine Überlegungen zu möglichen Relationen zwischen PH-Forschung und der strukturellen Valenzrealisierungstheorie. ${ }^{6}$ Als Ausgangspunkt bieten sich Überlegungen zu einer Gruppe von Partikelverben an (s. Ágel 2000, S. 142 ff.):

(1) Klaus montiert die Reifen an.

(1a) Klaus montiert die Reifen an das Auto.

(1b) Klaus montiert die Reifen an das Auto an.

Im Sinne der strukturellen Valenzrealisierungstheorie ist das Partikelverb anmontieren nicht zwei-, sondern dreiwertig. Doch während der Erst- und der Zweitaktant im strukturellen Normalfall makrorealisiert sind, d. h. Satzglieder im traditionellen Sinne darstellen, erscheint die kategorematische Form des Präpositionalobjekts mikrorealisiert, als Bestandteil der Verbform (Partikel an-). Der Unterschied zwischen (1) und (la) besteht darin, dass das Verb montieren - im Gegensatz zu anmontieren - keine Mikororealisierung des Drittaktanten zulässt. Das Präpositionalobjekt erscheint in (la) lediglich auf der Makroebene (an das Auto). Die Ausgliederung der Partikel an-aus der Verbform führt also ,zurück' zum Grundverb als VT. ${ }^{\text {? }}$

Typ (1b) stellt den Fall dar, in dem der Drittaktant als „Zwei-Ebenen-Aktant" (László 1988, S. 226) in Erscheinung tritt: Die Partikel an-wird auf der Makroebene als an das Auto expliziert. ${ }^{8}$

Wie ließe sich nun dieses Denkmodell auf PH anwenden?

Eine Verwendungsmöglichkeit der strukturellen Valenzrealisierungstheorie sehe ich darin, sie in die Bestimmung der morphosyntaktisch (Korhonen 1995) oder strukturell (Keil 1997, S. 67 ff.) genannten Variabilität mit einzubeziehen:

${ }^{6}$ Die strukturelle Valenzrealisierungstheorie untersucht die typologisch relevanten Merkmale der Aktantenrealisierung. Z.B. ist im lateinischen Satz Amo puellam der Erstaktant nur mikrovalenziell als Flexiv realisiert, während das Subjekt der deutschen Entsprechung Ich liebe das Mädchen primär makrovalenziell ist. In Abschnitt 4 möchte ich dafür argumentieren, dass sich die Idee der Unterscheidung zwischen Potenz und Realisierung einerseits und zwischen struktureller und kontextuell-situativer Realisierung andererseits auch auf die VT übertragen lässt.

${ }^{7}$ Die nicht gerade herkömmliche wortbildungsmorphologische Implikation dieser valenzrealisierungstheoretischen Interpretation ist, dass das Grundverb die makrostrukturelle Alternante des Partikelverbs darstellt.

8 Von der Explikation ist die Expansion zu unterscheiden, die nicht die normale, sondern eine besondere - diskursbedingte - Erweiterung der Mikrostruktur durch die Makrostruktur bedeutet, vgl. etwa Klaus montiert die Reifen an es an. Trotz Fischers Kritik, die die Partikel nur für eine Teilmikrorealisierung hält (Fischer 2003), möchte ich an der Interpretation der Partikel analog zu deiktischen Mikroflexiven festhalten. Berechtigt war dagegen der Kritikpunkt (ebd.), dass (1b) keine emphatische Makrorealisierung enthält, d.h. keine Expansion, sondern eine normale makrovalenzielle Explikation darstellt. 
(2) jmd. bindet jmdm. etwas auf

(2a) jmd. bindet jmdm. etwas auf die Nase

(2b) jmd. bindet/legt jmdm. etwas auf die Seele

(3) jmd. bringt jmdn. auf

(3a) jmd. bringt jmdn. auf die Palme

Soweit ich sehe, werden solche Fälle nicht als morphosyntaktische oder strukturelle Varianten - valenzträgertheoretisch gesprochen: Alternanten in Erwägung gezogen, obwohl man vielleicht behaupten könnte, dass auf die Nase und auf die Seele bzw. auf die Palme lexikalisierte Makroaktanten des lexikalisierten Mikroaktanten auf- des jeweils ersten Idioms sind. Dabei ist mir natürlich klar, dass diese Auffassung die ohnehin heikle Grenzfrage zwischen Wortbildung und Phraseologie in ein neues, aber für die Phraseologie vielleicht gar nicht unangenehmes Licht rückt: (eine bestimmte Sorte von) $\mathrm{PH}$ als Makrorealisierung einer (bestimmten Sorte von) Wortbildungskonstruktion oder umgekehrt: (eine bestimmte Sorte von) Partikelverb als Mikrorealisierung von (einer bestimmten Sorte von) $\mathrm{PH}$.

\section{Phraseologismus als (valenz)syntaktischer Normalfall}

Wie bereits angedeutet, bin ich der Ansicht, dass phraseologische Sprachzeichen nicht Sonderfälle, sondern den sprachlichen Normalfall repräsentieren. Insofern darf es auch keine , allgemeine ' Valenztheorie für , normale‘ Lexemwörter und getrennt davon eine ,besondere' Restvalenztheorie für polylexikalische Sprachzeichen geben. Was PH-Forschung und Valenztheorie m.E. gleichermaßen brauchen, ist ein in dreierlei Hinsicht integrativ angelegter Valenzansatz:

I. Der Ansatz soll die vier Grundfragen der Valenztheorie als empirisch gleich gewichtige Theorieelemente integrieren. ${ }^{9}$ Diese Art von Integrationsanspruch ist realistisch.

II. Der Ansatz soll eine VT-Theorie beinhalten, die zwar die empirischen Unterschiede zwischen phraseologischen und nichtphraseologischen Sprachzeichen erfasst, aber diese mit Hilfe desselben theoretischen Apparats beschreibt und erklärt. Diese Art von Integrationsanspruch ist wohl ebenfalls realistisch, aber im Moment noch programmatisch. Denn um sie einzulösen, bedarf es weiterer Forschungsaktivitäten und wohl auch gemeinsamer Forschungsprojekte.

III. Der Ansatz soll drei der vorhandenen vier Theorietypen des AP inte-

${ }^{9}$ Denn eine AP-Theorie ist noch weit - im Sinne von Tabelle 1 genau drei Theoriekomponenten weit - davon entfernt, eine Valenztheorie zu sein. Außerdem ist eine Valenztheorie mit einer AP-Theorie als zentraler Theoriekomponente per definitionem statisch wie jede Zuerst-System-dann-Gebrauch-Theorie (Ausnahmen wie Fischer 2001 bestätigen die Regel). Vermutlich braucht auch die PH-Forschung eher eine Valenztheorie mit gleichrangigen als eine mit nachrangigen Valenzrealisierungstheorien. 
grieren. Diese Art von Integrationsanspruch ist angesichts der zahlreichen relativ etablierten Theoriekonkurrenten zwar wohl weniger realistisch, aber m. E. notwendig.

$\mathrm{Da}$ der größte theoretische Nachholbedarf bei II besteht, konzentriere ich mich auf die Grundzüge einer integrativen VT-Theorie (Abschnitt 4.1). Da allerdings eine integrative VT-Theorie ohne eine integrative AP-Theorie fragmentarisch und auch schwer nachvollziehbar wäre, werden anschließend die Grundrisse einer integrativen AP-Theorie skizziert (Abschnitt 4.2). Abschließend soll das Zusammenspiel von VT- und AP-Theorie angedeutet werden (Abschnitt 4.3). ${ }^{10}$

\subsection{Grundzüge einer integrativen VT-Theorie}

Die am häufigsten und aus den verschiedensten Perspektiven erörterte valenztheoretische Frage in der PH-Forschung ist die der Unterscheidung zwischen interner und externer Valenz. Das Interesse ist kein Zufall, da die Frage alle in Abschnitt 1 genannten valenzbezogenen Themen der PH-Forschung tangiert. Entsprechend beachtlich sind auch die einschlägigen empirischen Forschungsergebnisse. ${ }^{11}$

Weniger befriedigend ist die theoretische Situation. Die Contradictio in Adjecto mit dem Begriff der internen Valenz kann an der frühen und bis heute zu Recht angesehenen Unterscheidung zwischen Konstruktionsmodellen und -gerüsten (Fix 1974/76 und 1979) gezeigt werden. Diese impliziert nämlich valenzträgertheoretisch, dass sich die interne Valenz nur dann als eine Art Komplementierung der externen begreifen lässt, wenn es sich um Konstruktionsmodelle handelt. Konstruktionsgerüste enthalten so etwas wie lexikalisierte interne Valenzstellen (z. B. die Stelle für außer Gefecht in jmdn. außer Gefecht setzen). Doch die Quelle einer Valenzstellenlexikalisierung könnte nur die Valenzpotenz des phraseologisch nicht gebundenen Verbs sein, was aber gerade bei Konstruktionsgerüsten nicht zutrifft $(* j m d n$. außer etw. setzen). Im Grunde würde also die, Anwendung' des Begriffs der internen Valenz auf die Konzeption von Fix implizieren, dass unikale interne Valenzstellen wie außer etw. in jmdn. außer Gefecht setzen Lexikalisierungen von phraseologisch nicht gebundenen Valenzstellen darstellen. Dabei schließt gerade die Unikalität interner Valenzstellen deren Zurückführung auf eine phraseologisch nicht gebundene Valenzstelle aus.

Darüber, dass der Begriff der internen Valenz „terminologisch nicht ganz

${ }^{10}$ Auf die im Vortrag kurz vorgestellte Möglichkeit, die vier Grundfragen zu integrieren $(=I)$, kann hier aus Raumgründen nicht eingegangen werden.

1 Nach Torzovas (1983) Auszählung stimmen bei 62\% der PH interne + externe Valenz mit der Valenz des phraseologisch nicht gebundenen Verbs überein. Auch der statistische Rest lässt sich relativ beruhigend erklären (s. Hessky 1988, S. 145), was aber nicht heißt, dass die interne Valenz immer als Restvalenz der externen ableitbar wäre (Hyvärinen 2003, S. 41, z. B. sich den Kopf über etw. zerbrechen). 
sauber" (Burger 1998, S. 21) ist, tröstet man sich damit hinweg, dass man betont, auf die Erkenntnisse, die man mit diesem problematischen Begriff verbindet, nicht verzichten zu können (ebd.; vgl. auch Hessky 1988, S. 141 f.).

Ich denke, dass es hier nicht darum geht, ob man auf Erkenntnisse verzichten will oder nicht. Niemand will das. Worum es geht, ist vielmehr, dass ein seit 30 Jahren ungelöstes Theorieproblem, das für PH-Forschung wie Valenztheorie zentral ist, im Interesse ,beider Seiten' ernst genommen und angepackt werden muss.

Wie könnte nun eine integrative VT-Theorie, mit der sich einerseits das Theorieproblem ,interne vs. externe Valenz' und andererseits die empirischen Probleme im Umkreis dieses Theorieproblems einer Lösung zuführen ließen, strukturiert werden?

Zwei Typen von Erkenntnissen in der PH-Forschung helfen bei der Lösung:

(1) Gottfried Kolde (1979, S. 82) hat darauf hingewiesen, dass die externe syntaktische Valenzpotenz eines $\mathrm{PH}$ von der intern gebundenen abhängt. Wenn z. B. eine NP im Nominativ bereits intern vorkommt (z. B. ein Stein in jmdm. fällt ein Stein vom Herzen), könne sie extern nicht mehr vorkommen.

(2) Nach Martina Keil (1997, S. 65 f.) ist die Grenze zwischen interner und externer Valenz nicht immer eindeutig zu ziehen, da es auch Übergangsfälle zwischen variablen und stabilen pronominalen Elementen von $\mathrm{PH}$ gebe. ${ }^{12}$

Demnach könnte der Schlüssel zur Lösung einerseits bei der empirisch intensiv untersuchten Variabilität phraseologischer VT liegen. ${ }^{13}$ Andererseits müsste der Binnenstruktur von $\mathrm{PH}$ theoretische Aufmerksamkeit gewidmet werden.

$\mathrm{Da}$ die Lösungsvorschläge in beiden Bereichen theoretischer ,Hinführungen' bedürfen, rechne ich mit insgesamt vier Bausteinen einer integrativen VT-Theorie. Diese sind:

1. ein Stufenmodell der Formvariabilität;

2. im Anschluss an dieses eine VT-Theorie, in der einerseits offene und geschlossene VT, andererseits potenzielle und (strukturell bzw. kontextuell-situativ) realisierte VT unterschieden werden;

${ }_{12}$ Auch die Forschungsergebnisse zur semantischen Autonomie von Idiomkonstituenten wie etwa die Irregularitätsmerkmale von Dobrovol'skij (1995, S. 27 ff.) legen einen skalaren Übergang zwischen interner und externer Valenz nahe.

13 Wenn man bedenkt, dass PH (im weiteren Sinne) mit den Merkmalen ,Polylexikalität ${ }^{*}$ und ,Festigkeit' beschrieben werden (Burger 1998, S. 14 f.), fällt auch hier das gewaltige Theoriedefizit auf. Denn die Breite der empirischen Variabilität (s. etwa Korhonen 1995) ist mit den gebetsmühlenartig wiederholten Bekenntnissen zur ,Relativität ${ }^{\star}$ der Stabilität theoretisch nicht in den Griff zu bekommen. Auch die Statistik der erfahrenen Lexikographin Rosamund Moon spricht Klartext: $40 \%$ der PH in dem 18 Millionen Wortformen enthaltenden Hector Korpus (für Britisches Englisch) haben keine ,frozen or fixed canonical forms, and I believe this figure will be the same or even higher in larger corpora" (Moon 1998, S. 92). 
3. eine Aktantenhierarchie;

4. im Anschluss an diese ein Modell der Binnenstruktur von $\mathrm{PH}$.

Zu 1-2:

Die folgende Darstellung zeigt eine erste Überlegung zu einem Stufenmodell der Formvariabilität: ${ }^{14}$

Darstellung 1: Stufenmodell der Formvariabilität

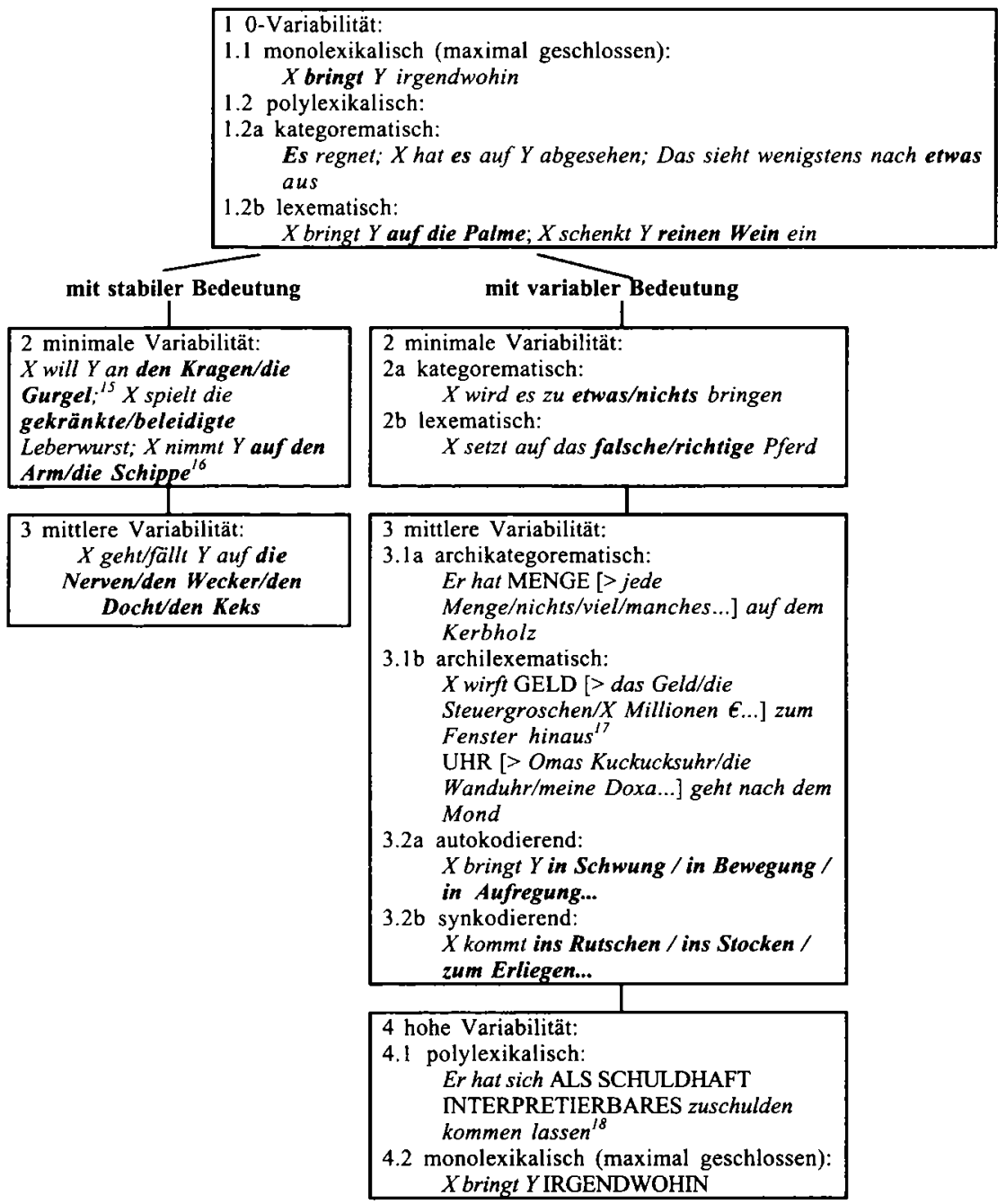

14 In den Beispielen, die den einzelnen Klassen zugeordnet wurden, ist der Teil, auf den sich die Bezeichnungen der Unterklassen beziehen, immer hervorgehoben. Dabei handelt es sich entweder um Sprachzeichen (halbfett gesetzt) oder um Metasprachliches (in Versalien). 
Worauf es hier ankommt, ist nicht die Qualität, also nicht die Frage, ob es sich um lexikalische oder strukturelle Varianten handelt, und auch nicht die Frage, wie man die Grenze zwischen lexikalischer Variation und Synonymie zieht. Es geht hier ausschließlich um die Variationsbreite, wobei allerdings eine Rolle spielt, ob diese mit Bedeutungsvariation einhergeht (rechte Spalte) oder nicht (linke Spalte). ${ }^{19}$

Die nähere Erläuterung des Stufenmodells lässt sich mit der Vorstellung des zweiten Bausteins der angekündigten VT-Theorie verbinden:

Ein maximal geschlossener VT ist ein freies (= phraseologisch nicht gebundenes) Verb, eine monolexikalische Formganzheit. Es ist, wohlgemerkt, ein Extremfall (Stufe 1.1). In dem Augenblick, wo polylexikalische Formganzheiten im Spiel sind, besteht nämlich die Möglichkeit, den VT skalar zu öffnen. Wenn diese Möglichkeit nicht genutzt wird oder nicht genutzt werden kann, bleibt allerdings auch der polylexikalische VT geschlossen (Stufe 1.2). In beiden Fällen geht es um 0-Variabilität. ${ }^{20}$

Den minimalen Grad der Öffnung stellen die Alternativfälle dar (Stufe 2). Der VT, der hier zwei Formvarianten hat, ist zwar nicht geöffnet, kann aber bei spielerisch-kreativer Modifikation geöffnet werden: Es ist ein geschlossener, aber kein hermetisch verschlossener VT.

Valenzträgertheoretisch und aus der Sicht des intern/extern-Problems besonders bedeutsam sind die Fälle mittlerer Formvariabilität (Stufe 3). Hier geht es um Reihenbildungen, wobei diese die referenzielle Bedeutung des $\mathrm{PH}$ entweder unberührt lassen (stabile Bedeutung) oder nicht (variable Bedeutung). Im letzteren Falle handelt es sich entweder um eine im Rahmen einer archikategorematischen oder archilexematischen Vorgabe eingeschränkte Bedeutungsvariation (3.1) oder um eine prinzipiell uneingeschränkte (3.2: FVG) mit ebenfalls zwei Untergruppen: autokodierend oder nicht (= synkodierend). ${ }^{21}$

${ }^{15}$ Nach Burger (1998, S. 26) sollte man hier von zwei verschiedenen PH sprechen.

${ }^{16}$ Im Gegensatz zu Gurgel/Kragen sind Schippe/Arm keine, referenziellen'Synonyme.

${ }^{17}$ Hierher gehört auch jmdm. leicht, glatt, schwer o. ä. von der Z. gehen. Nach Korhonen (1988, S. 108) liegen hier modale Adverbiale außerhalb des Idioms vor. Als ,eine adäquate lexikographische Form" (ebd.) biete sich daher in bestimmter Weise an (jmdm. in bestimmter Weise von der Zunge gehen oder mit jmdm. in bestimmter Weise ins Gericht gehen).

${ }^{18}$ Beispiele für ganz normale Slot-Füllungen in COSMAS sind u. a. eine Ehrlosigkeit, das Versäumnis, persönliche Übergriffe, eine Menge, leichtfertige Äußerungen, Übertretungen, ein Verkehrsvergehen, Fehler.

${ }^{19}$ Die Beschreibung der Stufen der Bedeutungsvariation erfolgt in loser Anlehnung an Coserius Modell der lexikalischen Solidaritäten mit den ,zunehmend' variablen Solidaritätstypen der Implikation, Selektion und Affinität (Coseriu 1967, S. 299).

${ }^{20}$ Polylexikalische 0 -Variabilität ist allerdings einen Hauch offener als die 0 -Variabilität von freien Verben, da sich phraseologisch gebundene Konstituenten an grammatischen Prozessen (Flexion, Serialisierung, u. U. Passivierung usw.) beteiligen. Und innerhalb von Stufe 1.2 ist der lexematische Typus offener als der kategorematische, der weniger spielerisch-kreative Modifikation erlauben dürfte.

21 Den Begriff der Autokodierung (= autonomen Kodierung) verwende ich in Anlehnung 
Mittlere Formvariabilität interpretiere ich valenzträgertheoretisch als den Typus, bei dem eine pauschale Zuordnung zur internen oder zur externen Valenz zu kurz greifen würde. Vielmehr geht es hier um offene VT, d. h. um VT mit einem Slot. Der Slot ist derjenige Bestandteil des VT, der obligatorisch aufzufüllen ist, damit ein VT als VT überhaupt funktionstüchtig wird. Im Unterschied zu Leerstellen gestalten Slots die Binnenstruktur von PH mit.

Die Idee des offenen VT möchte ich ausgehend von zwei COSMAS-Belegen kurz erläutern:

(3) Was die Agrargemeinschaft besonders auf die Palme bringt, ist die Tatsache, dass man vom Land Steiermark bei der Abwicklung der rechtlichen Verfahren nicht einmal eingeladen wurde.

(Kleine Zeitung, 24.08 1999; Baustopp-Antrag bei Riesneralm-Ausbau)

(4) Das Gelände in unmittelbarer Nähe zur Innenstadt, auf dem einst das Hallenbad stand, bringt offensichtlich die Phantasie in Bewegung [...]. (Frankfurter Rundschau, 27.07 1998; Diskussion um die Zukunft Hofheims am FR-Mobil)

Im Gegensatz zur 0- bzw. zur minimalen Variabilität, bei denen der potenzielle und der strukturell realisierte VT in einer ,linearen“ Type-Token-Relation stehen, besteht bei einem VT mit einem Slot ein ,materieller' Unterschied zwischen potenziellem und strukturell realisiertem VT. Während also das Token auf die Palme bringt in (3) eine Realisierung des Types auf die Palme bringen ist, wird angenommen, dass der realisierte VT bringt in Bewegung in (4) durch die aktuelle, strukturell obligatorische Auffüllung des autokodie-

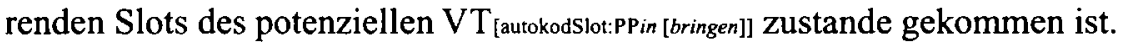

Entsprechend den fünf grundlegenden Möglichkeiten mittlerer Variabilität ( 1 mit stabiler, 4 mit variabler Bedeutung) gehe ich davon aus, dass es auch fünf Typen von Slots gibt: einen referenzerhaltenden Typ und vier referenzauffüllende Typen: archikategorematischer, archilexematischer, autokodierender und synkodierender Slot. Der theoretische Unterschied zwischen dem referenzerhaltenden Typ und den referenzauffüllenden Typen besteht darin, dass bei letzteren erst die referenzielle Auffüllung der Slots zur Festlegung der Prädikation führt. Somit besteht hier ein ausgeprägter Unterschied zwischen dem Prädikationspotenzial des potenziellen und der Prädikation des strukturell realisierten VT.

Am Ende der Skala der Formvariabilität stehen Fälle mit hoher Variabilität (Stufe 4), d.h. Leerstellen (z. B. die Stelle für die Direktional-E in 4.2). Hier schließt sich der Kreis zur 0-Variabilität, da die Formstellen mit hoher

an Zifonun/IdS-Grammatik (1997, S. 1039 ff.). Eine PP wie in Bewegung kodiert eine autonome satzsemantische Information unabhängig davon, ob sie sich in einem gegebenen Kontext etwa mit setzen, sich setzen, bringen, sein, geraten, kommen, halten, umsetzen, versetzen usw, verbindet. Demgegenüber kodiert etwa die PP ins Rutschen nur gemeinsam mit ihrem jeweiligen Kontext eine satzsemantische Infomation, ist also synkodierend. 
Variabilität die Grenze des VT von außen markieren..$^{22}$ Auch hier handelt es sich also um geschlossene $\mathrm{VT}^{23}$

Zu 3-4:

Obwohl bereits Tesnière von Erst-, Zweit- und Drittaktanten gesprochen und somit eine Aktantenhierarchie suggeriert hatte, blieben die meisten Valenztheorien und Dependenzgrammatiken bei der Gleichstellung des Subjekts mit den Objekten, obwohl es jedermann klar war, dass die einzelnen Aktanten wohl unterscheidbare grammatische und semantische Eigenschaften haben. ${ }^{24}$

Der Punkt, der uns im Moment interessiert und über den sich übrigens wohl alle Grammatiktheorien einig sind, ist jedoch die ,Sonderstellung' jedes einzelnen Aktanten gegenüber jedem anderen Aktanten. Denn es gibt den „einheitlichen Block der Objekte gegenüber dem Subjekt [...]" auch nicht (Eisenberg 1994, S. 285).

Die ,Sonderstellungen' der einzelnen Aktanten sind aber nicht atomistisch zu denken. Denn zu Recht wird in den Grammatiktheorien ebenfalls angenommen, dass es eine Aktantenhierarchie - eine Hierarchie der Argumentanbindung - gibt, die eine Art Verbnähe reflektiert. Um welche Art Verbnähe es dabei geht und wie die Hierarchie ausbuchstabiert werden kann, darüber gehen die Auffassungen auseinander (vgl. den Überblick in Welke 2002, S. $92 \mathrm{ff}$.).

Ich nehme in Anlehnung an Welke (1994 und 2002, S. 103 ff.) an, dass die Aktanten eines VT den entworfenen Sachverhalt inhärent perspektivieren, dass es also bei jedem VT eine Aktantenhierarchie gibt, die die logisch-pragmatische Rangfolge der Beteiligtenrollen (= Argumente) widerspiegelt. Die Hierarchie manifestiert sich in der unmarkierten Nebensatzwortstellung:

(5) ... dass Egon (1.Arg.) mir (2.Arg.) das Buch (3.Arg.) gibt.

(Beispiel nach Welke 2002, S. 106)

Die Aktantenhierarchie spielt valenzträgertheoretisch solange keine nennenswerte Rolle, solange der VT monolexikalisch ist. In diesem Falle besteht nämlich die Binnenstruktur des VT aus der bloßen Verbkategorie. Man vergleiche aber (5) mit (6):

(6) ... dass Egon (1.Arg.) mir (2.Arg.) die Leviten (3.Arg.) liest.

Binnenstruktur: die Leviten [lesen] oder 3.Arg.: NPakk $\left[\mathrm{V}_{\mathrm{FosP}}\right]$

${ }^{22}$ In einem Fall wie $X$ bringt $Y$ irgendwohin wird also die Grenze des VT durch die Monolexikalität des VT gewissermaßen von innen (Stufe 1.1) und durch die Direktionalstelle von außen (Stufe 4.2) markiert.

${ }^{23}$ Auch hier gilt, dass monolexikalische Geschlossenheit einen Hauch geschlossener ist als polylexikalische.

${ }^{24}$ Der Grund für diese Haltung war und ist m. E., dass man die Frage der Sonderstellung des Subjekts (gegenüber den Objekten) mit der der Gleichstellung des Subjekts (mit dem Prädikat) verwechselt (zur Problematik s. ausführlich Ágel 2000, S. 83 ff.). 
Hier ist das dritte Argument im VT gebunden, was zur Konsequenz hat, dass nur das erste und das zweite Argument als Leerstellen in Frage kommen. Die Binnenstruktur des VT die Leviten lesen lässt sich mit dem dritten Argument als Operanden und dem formspezifisch agierenden Verb als Operator beschreiben..$^{25}$ Es geht also nicht bloß darum, dass der polylexikalische VT formal indizierbare Konstituenten hat, sondern auch darum, dass die Binnenstruktur des VT hierarchisch ist, was sich aus der Aktantenhierarchie ableiten lässt. Mithin geht es auch darum, dass der VT nicht nur eine externe Kategorie hat (etwa: intransitives Verb), sondern auch darum, wie diese Kategorie intern, zustande kommt'. Denn nur aus der externen Kategorie und aus der internen Strukturierung zusammen lassen sich die freien Satzbildungspotenzen ableiten. Beispielsweise sind die $\mathrm{PH}$ die Leviten lesen und sich zuschulden kommen lassen beide intransitiv (externe Kategorie), aber nur der letztere PH lässt keine Dativstelle zu, da hier das 2. Argument (sich) intern bereits gebunden ist.

Damit sind wir gleich bei dem vierten Baustein, der Modellierung der Binnenstruktur von PH im Anschluss an die Aktantenhierarchie, angekommen. In Tabelle 2 (siehe nächste Seite) wird versucht, einige VT-Grundtypen darzustellen und kategorial zu beschreiben. ${ }^{26}$

Die Darstellung beschränkt sich - mit Ausnahme des Sprichworts am Ende - auf Verben mit drei Argumenten. Sie fängt mit einem Simplex - einem monolexikalischen VT - mit drei Leerstellen an und endet mit zwei (verschiedenen) Typen von polylexikalischen VT, die keine Leerstellen mehr und somit auch keine valenzpotenziell generierbare Satzbildungspotenz haben. Doch selbst bei diesen gewöhnlich als satzwertig, d.h. durch die externe Kategorie ,Satz', charakterisierten PH, spielt bei Modifikationen die Binnenstruktur eine Rolle:

(7) Ich frage mich, ob nur mir aller Anfang schwer ist.

(8) *Ich frage mich, ob ihm das dem Fass den Boden ausschlägt.

Da das Sprichwort kein im VT gebundenes zweites Argument hat, kann es durch einen freien Dativ erweitert werden (s. (7)). Dagegen ist diese Möglichkeit bei der festen Phrase bereits ausgeschöpft (s. (8)). ${ }^{27}$

Wie erwähnt besteht bei referenzauffuillenden offenen VT ein Unterschied zwischen dem Prädikationspotenzial des potenziellen und der Prädikation des

${ }^{25}$ Formspezifik (= FOSP) - in etwa vergleichbar mit den traditionellen Begriffen der (formalen) Rektion bzw. der syntaktischen Valenz - gilt als eine Valenzrelation (s. auch Abschnitt 4.2 unten).

26 Abkürzungen: makro/mikro (PP) = makrovalenziell/mikrovalenziell realisiert(e PP) (s. die Beispiele (3) und (3a) in Abschnitt 3); autokodSlot/synkodSlot/archilexSlot = autokodierender Slot/synkodierender Slot/archilexematischer Slot. Die Abkürzung $P P$ zum ist kein Tippfehler, sondern eine Verlegenheitslösung, mit der die Fixiertheit der Verschmelzung angezeigt werden soll.

27 Selbstverständlich ist kreativ-wortspielerische Modifikation etwa durch Einfügung von Angaben oder Attributen jederzeit möglich. 
Tabelle 2: Binnenstruktur von phraseologischen Valenzträgern

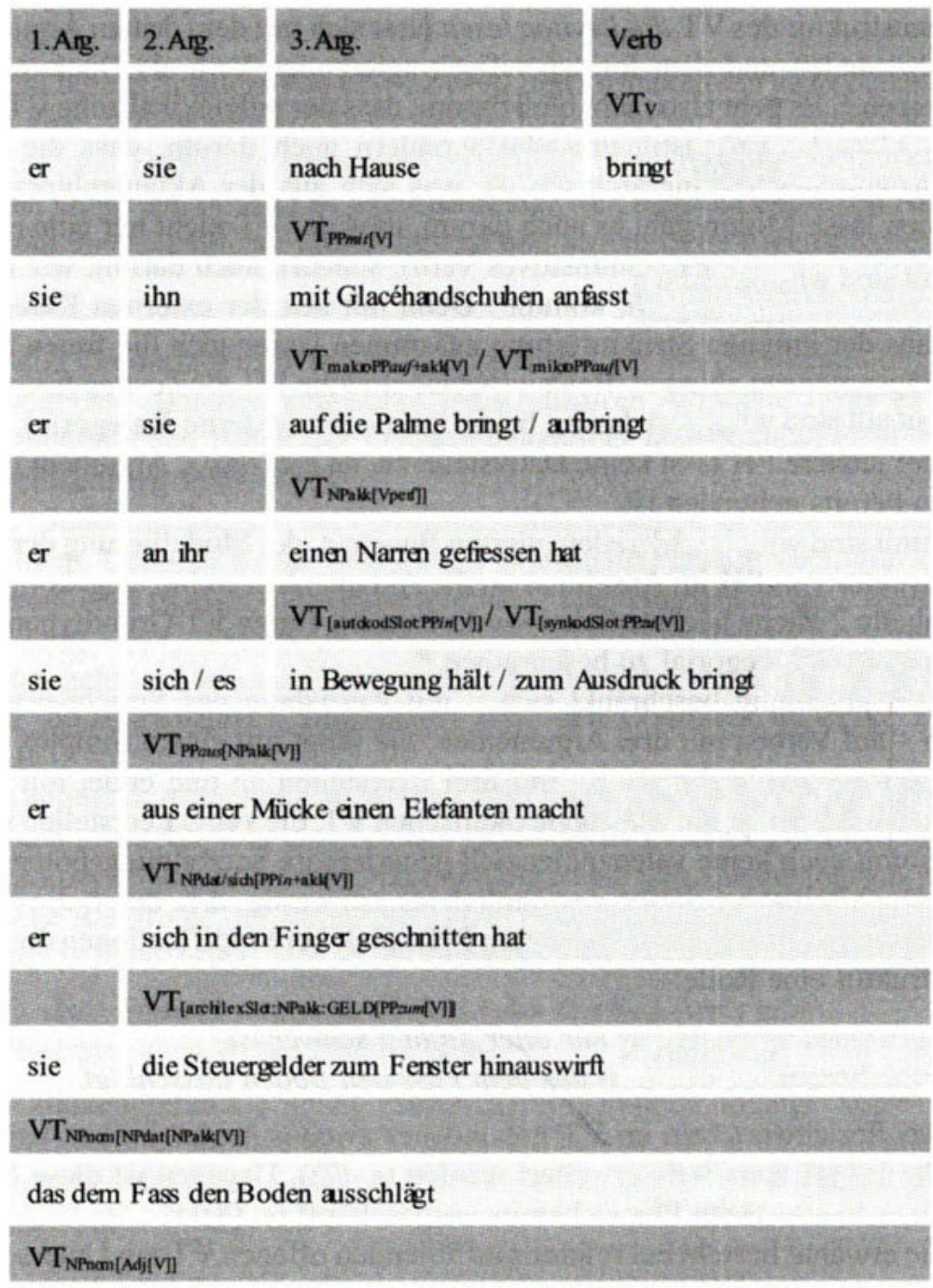

alle Anfang schwer ist

strukturell realisierten VT: Erst die referenzielle Auffüllung des Slots führt zur Festlegung der Prädikation des realisierten VT. Wenn dabei der nichtverbale VT-Bestandteil semantisch relativ autonom ist, wenn also Kompositionalität oder wenigstens Teilbarkeit/Isomorphie zwischen der formalen und semantischen Struktur (im Sinne von Dobrovol'skij 1995, S. 27 ff.) vorliegt, kann die Slot-Auffüllung syntaktisch und somit auch referenziell weiter, ausgebaut' werden. Das Ergebnis ist ein mitunter recht umfangreicher VT: 
(9) Was Hitler übersah, war die Tatsache, daß die tiefen ideologischen Gegensätze zwischen den Angelsachsen und den Russen nicht zum Austrag, schon gar nicht zum kriegerischen Austrag kommen konnten, solange Deutschland [...] gewissermaßen als Isolierschicht zwischen ihnen stand.

(Sebastian Haffner: Von Bismarck zu Hitler. München, S. 299)

Somit liegt es theoretisch nahe, einen VT auf drei Abstraktionsebenen zu erfassen: ${ }^{28}$

(1) als potenzieller VT, hier: [synkodSlot:PP $\mathrm{P}_{z u}[$ kommen]];

(2) als strukturell realisierter VT, hier: zum Austrag kommen;

(3) als kontextuell-situativ realisierter VT: nicht zum Austrag, schon gar nicht zum kriegerischen Austrag kommen konnten.

Die Darstellung in Tabelle 2 ist natürlich erst ein Anfang. Sie reflektiert mindestens viererlei nicht:

(a) den Umgang mit gebundenen Angaben (z. B. die $\mathrm{PP}_{\text {mit }}$ in mit einem Fuß im Grabe stehen);

(b) die Problematik ,besonderer' Leerstellen (s. Abschnitt 2);

(c) die strukturell-typologische und

(d) die Markiertheitsproblematik der Leerstellen.

Was (b) anbelangt, könnte in Anlehnung an Sadziński (1989) zwischen primären - bei ihm: statischen - und sekundären - bei ihm: dynamischen - Aktanten, die aus primären Angaben hervorgehen, unterschieden werden. ,Besondere' Leerstellen wie etwa die $\mathrm{PP}_{\text {furr }}$ in eine Antenne für etw. haben wären dann sekundäre Aktanten und daher auf einer (noch zu etablierenden) sekundären Ebene der Aktantenklassifikation zu erfassen.

Was (c) anbelangt, ist die Trennung zwischen absolutiven VT (mir brummt/schwirrt der Kopf; ihm rutscht die Hand aus) und nominativen VT (alle Beispiele in Tabelle 2) notwendig, weil das zwei verschiedene (typologische) Typen von Aktantenhierarchien sind.

Schließlich - (d) - wäre noch zu überlegen, ob aus unmarkierten Valenzen synthetisierte VT (z.B. die Leviten lesen) getrennt von aus markierten Valenzen (Blume 2000) synthetisierten VT (z. B. mit dem Zaunpfahl winken) zu beschreiben sind.

${ }^{28}$ Dabei kann ein ,materieller' Unterschied zwischen allen drei Ebenen nur bei offenen VT vorliegen. Bei geschlossenen VT beschränkt sich diese Möglichkeit auf den Unterschied zwischen potenziellem und strukturell realisiertem VT einerseits (z.B. auf die Palme bringen) und kontextuell-situativem VT (z. B. Was X auf die berühmte Palme gebracht hat, ist ...) andererseits. 


\subsection{Grundzüge einer integrativen AP-Theorie}

Die aktuell konkurrierenden AP-Theorien lassen sich in vier Typen einteilen (ausführlicher s. Ágel 2000, S. 199 ff.):

Tabelle 3: Theorien des Aktantenpotenzials

$\begin{array}{ll}\text { (I) Dichotomische Theorien des AP } & \text { (II) Zentrum/Peripherie-Theorien des AP }\end{array}$

(1) multidimensional (3) multidimensional (= prototypisch)

(2) eindimensional $\quad$ (4) eindimensional (= graduell)

Während dichotomische Theorien - trotz eventueller ,Grauzonen' - mit einer grundsätzlichen E/A-Unterscheidbarkeit rechnen, gehen Zentrum/ Peripherie-Theorien davon aus, dass E/A-Grenzen entweder höchstens nur in Bezug auf einzelne Valenzrelationen oder gar nicht anzunehmen sind.

Um die Unterschiede zwischen den vier Typen anzudeuten, wird in vereinfachter und vereinheitlichter Darstellungsform gezeigt, wie ein Vertreter des jeweiligen Theorietyps die NPn in (10) und (11) interpretieren würde: ${ }^{29}$

(10) Heß legte einen zweiten Obduktionsbefund sowie die eidesstattliche Erklärung eines südafrikanischen Geheimdienstoffiziers vor, der (NP $)$ zwei britische Agenten $\left(\mathrm{NP}_{2}\right)$ des Mordes an Heß $\left(\mathrm{NP}_{3}\right)$ beschuldige. (Mannheimer Morgen, 17.08 1989, Politik; Sohn von Rudolf Heß wiederholt Mordtheorie)

(11) Es kommt schon einmal vor, daß ich ( $\left.\mathrm{NP}_{1}\right)$ jemanden $\left(\mathrm{NP}_{2}\right)$ zu Unrecht beschuldige.

(Neue Kronen-Zeitung, 31.03 1996, S. 28; Was bedeutet für Sie Ehrlichkeit?)

(1) Engel: SUBKLASS (VT, $\left.\left(\mathrm{NP}_{1}, \mathrm{NP}_{2}, \mathrm{NP}_{3}\right)\right)$ \& NOT (VT, $\left.\left(\mathrm{NP}_{1}, \mathrm{NP}_{2}\right)\right)$

(2) Fischer: BET (VT, $\left(\mathrm{NP}_{1}, \mathrm{NP}_{2}, \mathrm{NP}_{3}\right)$ )

(3) Jacobs: (FOSP \& INSP \& ARG (VT, $\left.\left(\mathrm{NP}_{1}, \mathrm{NP}_{2}, \mathrm{NP}_{3}\right)\right)$ ) \& NOT (VT, $\left(\mathrm{NP}_{1}, \mathrm{NP}_{2}\right)$ )

(4) Heringer: PRÄSUPP (VT, NP2) > PRÄSUPP (VT, NP 1$)>$ PRÄSUPP (VT, $\mathrm{NP}_{3}$ )

${ }^{29}$ Die Vereinheitlichung der Bezeichnungen für die einzelnen Valenzrelationen erfolgt mit Ausnahme von PRÄSUPP in Anlehnung an Jacobs (1994, S. $14 \mathrm{ff}$.): SUBKLASS = Subklassenspezifik; NOT = Notwendigkeit; BET = Beteiligtheit (oder Sachverhaltsbeteiligung); FOSP = formale Spezifizität; INSP = inhaltliche Spezifizität; ARG = Argumenthaftigkeit. PRÄSUPP (Ágel 2000, S. 209) steht für ASSOZ (= Assoziiertheit) bei Jacobs (1994, S. 29). Ausführlich besprochen werden die einzelnen Relationen in Ágel 2000, S. $171 \mathrm{ff}$. 
Typ (1) ist aus theoretischen und methodischen Gründen nicht mehr vertretbar. ${ }^{30}$ Wohlgemerkt, es handelt sich um den Theorietyp, der das ,Valenzden-

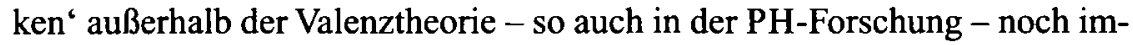
mer beherrscht.

Eine integrative AP-Theorie müsste sich demnach auf die restlichen drei Theorietypen stützen:

Zwischen den Theorietypen (2) und (3) besteht eine Art philosophischer Unterschied. Nach dem eindimensional-dichotomischen Theorietyp ist Valenz ein unhintergehbares Grundphänomen, während sie nach dem die Theoriediskussion aktuell dominierenden prototypischen Theorietyp ein Epiphänomen darstellt.

Was nun den graduellen Theorietyp anbelangt, er lässt sich nicht in das Prokrustesbett des Entweder-Grund-oder-Epiphänomens hineinzwängen, da es sich hier um eine kognitiv-psychologische Dynamisierung von traditionell eher statischen valenztheoretischen Positionen handelt. Theorietyp (4) ist also auf einer anderen begriffslogischen Ebene anzusiedeln als die Typen (2) und (3). Doch auch die Theorietypen (2) und (3) ließen sich durchaus als begriffslogisch disloziert begreifen, als Theorietypen nämlich, die das Valenz genannte Phänomen von verschiedenen Perspektiven aus zu verstehen trachten: Theorietyp (2) synthetisch, im Geiste von Tesnières Dependenzgrammatik, Theorietyp (3) analytisch, im Geiste formallogischer und -linguistischer Traditionen.

Wenn nun die These von der begriffslogischen Dislokation der Theorietypen (2), (3) und (4) haltbar ist, so besteht vielleicht die Möglichkeit, sie in einer Gesamttheorie des AP zu integrieren. Das Verhältnis der drei Theorietypen in einer integrativen AP-Theorie könnte wie folgt aussehen:

1. Insofern VT in der Lage sind, Sachverhalte kontext- und situationsbezogen zu entwerfen (Fischer 2001), sind sie BET-VT. Die einzige synthetische, d.h. andere Relationen zu einer kognitiven Einheit zusammenfügende, Valenzrelation besteht zwischen einem BET-VT und seinen aktuell konstituierten Sachverhaltsbeteiligten (= synthetischen Aktanten/BET-Aktanten).

2. Insofern potenzielle VT ihre BET-Umgebung inhaltlich und/oder formal prädeterminieren, sind sie INSP- und/oder FOSP-VT. INSP und FOSP stellen analytische Valenzrelationen dar.

3. Was die ebenfalls, klassische' Relation NOT anbelangt, es gibt im Grunde zwei extreme Herangehensweisen an sie: Entweder man überlässt sie ganz dem Kontext und der Situation (traditionelle o/f-Unterscheidung) oder man gliedert sie ganz in das Aktantenpotenzial ein (Jacobs 1994a). Ich denke, dass ein integrativer Valenzansatz die Möglichkeit bietet, zwischen diesen zwei

${ }^{30}$ Theoretisch geht es vor allem um die Probleme mit einer Valenzrelation SUBKLASS, methodisch um die Einebnung der Unterschiede zwischen den Valenzrelationen SUBKLASS und NOT (s. Ágel 2000, S. 187 ff. und S. 197 f.). 
Herangehensweisen zu vermitteln. Wenn man nämlich den Blick nicht nur auf die getrennte Betrachtung der analytischen Valenzrelationen richtet, sondern auch auf deren Integration in Valenzrealisierungsmustern, so wird es klar, dass NOT nicht modular - auf einzelnen Aktanten oder Relationen -, sondern global - auf (den analytischen Valenzrelationen basierenden) Valenzrealisierungsmustern - operiert. ${ }^{31}$ Der Frage der Notwendigkeit eines, inhaltlosen ${ }^{*}$ FOSP-Aktanten oder eines, formlosen' INSP-Aktanten ist weder empirische noch theoretische Relevanz beizumessen. ${ }^{32}$ Infolgedessen soll NOT als ein Valenzrealisierungsoperator bestimmt werden, der auf den FOSP-INSP-Realisierungsmustern operierend die analytische Bestimmung der Valenzrealisierungsmuster abrundet. Valenzrealisierungsmuster ( $=$ valenzgenerierte ,Satzbaupläne') sind demnach als NOT(INSP-FOSP)-Realisierungsmuster zu definieren. Sie enthalten INSP- und/oder FOSP-Stellen, deren ,Bündelung' zu einem bestimmten Modell von NOT reguliert wird. Beispielsweise ist im Falle von beschuldigen (s. die Belege (10) und (11) oben) mit zwei Mustern zu rechnen: SU[DO[GEN-O[V]]] (Beleg (10)) und SU[DO[[V]] (Beleg (11)). ${ }^{33}$ Welches von diesen im Onlinediskurs realisiert wird, hängt in hohem Maße von der Valenzrelation PRÄSUPP ab (Heringer 1984 und 1985).

4. Damit sind wir bei den Fragen angekommen, welche Art von Valenzrelation PRÄSUPP ist bzw. wie sich der graduelle Theorietyp integrieren lässt. Ich denke, PRÄSUPP erhält valenztheoretische Relevanz, wenn sie nicht auf einzelne Sachverhaltsbeteiligte oder -nichtbeteiligte bezogen wird, sondern als eine kognitiv-psychologische Relation zwischen BET-VT und Valenzrealisierungsmustern aufgefasst wird. PRÄSUPP ist ein Modell des sog. assoziativen Primings (Gansel 2003, S. 14) zwischen zwei Wissensstrukturen: zwischen lexematischem Wissen und Schemawissen. Ob im Falle einer Aktivierung von beschuldigen das Schemawissen SU[DO[GENO[V]]] (Beleg (10)) oder SU[DO[[V]] (Beleg (11)) mit aktiviert wird, hängt in hohem Maße davon ab, wie stark die einzelnen Schemata im aktuellen Wissensbestand des aktuellen Sprechers/Schreibers präsupponiert sind. Im Gegensatz zu den eher statischen Relationen INSP, FOSP und NOT ist PRÄSUPP - zusammen mit der im Sinne von Fischer (2001) interpretierten BET - als eine dynamische Valenzrelation zu bestimmen, die im Onlinediskurs mit dafür sorgt, dass die eher statischen Valenzrelationen aktiviert werden.

${ }^{31}$ Ich ziehe die Bezeichnung, Valenzrealisierungsmuster" traditionellen Termini wie ,Satzbauplan“ oder ,Satzmodell' nicht nur aus Gründen der terminologischen Kohärenz vor, sondern auch deshalb, weil ich meine, dass zwischen den Stellen eines ,Satzbauplans' keine ,flache` Beziehung besteht (s. Abschnitt 4.3).

32 Und was die synthetische BET-Relation anbelangt, auf sie lässt sich erst recht nicht die NOT-Relation anwenden, da sich Sachverhaltsbeteiligte per definitionem an dem Entwerfen eines Sachverhalts beteiligen müssen.

${ }^{33} \mathrm{SU}=$ Subjekt; DO = Direktes Objekt; GEN-O = Genitivobjekt. Zur Interpretation der Klammerung s. Abschnitt 4.3. 


\subsection{Das Zusammenspiel von Valenzträger und Valenzrelationen}

Ein Plädoyer für Aktantenhierarchien (Abschnitt 4.1) impliziert, dass man annehmen muss, dass Valenzrealisierungsmuster - im Gegensatz zu den wohl bekannten Satzbauplänen - entsprechend den in der gegebenen Einzelsprache vorhandenen Typen von Aktantenhierarchien ebenfalls über eine Binnenstruktur verfuigen. Im Unterschied etwa zu SU[DO[[V]] stellt demnach SU-DO-V m. E. kein aktivierbares Satzschema des Deutschen dar. Die Klammerungen indizieren eben die Bestimmungsrelationen.

Das Postulat von Binnenstrukturen für VT wie für Valenzrealisierungsmuster ist eine wichtige Voraussetzung der Modellierbarkeit des Zusammenspiels von VT und Valenzrelationen. Ich möchte es ausgehend von einer strukturellen und einer kontextuell-situativen Realisierung des potenziellen VT auf die Palme bringen vorstellen:

(3) Was die Agrargemeinschaft besonders auf die Palme bringt, ist die Tatsache, dass man vom Land Steiermark bei der Abwicklung der rechtlichen Verfahren nicht einmal eingeladen wurde.

(Kleine Zeitung, 24.08 1999; Baustopp-Antrag bei Riesneralm-Ausbau)

(13) Das Schneckentempo auf der „Maruta Jaya“ ist Schenzle ein Greuel. "Wir haben keine Eile“, ist der Satz, der den Hamburger Forscher auf die indonesische Palme bringt und aus geplanten vier 18 Jahre Projektdauer machte. „Maruta Jaya “ ist nicht das einzige Beispiel für alternativen Antrieb.

(Neue Kronen-Zeitung, 10.08 1997, S. 14; $\left.{ }^{*}\right)^{34}$

BET-VT verfügen über eine PRÄSUPP-Potenz, deren Aktivation zu der assoziativen,Zündung ' eines Valenzrealisierungsmusters führt. Im Falle von (3) und (13) können wir mit der Aktivation von SU[DO[PO $\left.\left.{ }_{a u f+a k k}[\mathrm{~V}]\right]\right]$ rechnen. ${ }^{35}$

Ein ,Zünden ' bedeutet allerdings über die Aktivierung hinaus, dass die Binnenstruktur des PH mit der des aktivierten Valenzrealisierungmusters abgeglichen wird. Die Binnenstruktur des VT auf die Palme bringen ist ,makroPPauftakk[V]" (s. Tabelle 2 in Abschnitt 4.1). Die Abgleichung kann grundsätzlich dreierlei bedeuten:

(1) Die Argumentstellen des Valenzrealisierungsmusters, die in der Binnenstruktur des PH nicht vorhanden sind, werden geöffnet. Dies betrifft in (3) und (13) jeweils das 1. (= SU) und das 2. Argument (= DO).

(2) Die Argumentstellen des Valenzrealisierungsmusters, die in der Binnenstruktur des PH vorhanden sind, werden geschlossen oder freigesetzt. Dies betrifft in (3) und (13) jeweils das 3. Argument (= POauftakk). Wenn sie geschlossen werden wie in (3), wird nur die phraseologische Bedeu-

${ }^{34}$ Das Sternchen ist Teil der COSMAS-Belegstellenangabe. Vermutlich handelt es sich dabei um einen Fehler.

${ }^{35} \mathrm{PO}=$ Präpositionalobjekt. 
tung realisiert. Denn ,eine Stelle schließen' heißt, diese für weitere syntaktische Operationen nicht mehr freizugeben.

(3) Dagegen bedeutet die durch den Onlinediskurs induzierte Freisetzung des 3 . Arguments wie in (13) die syntaktische Reaktivierung des gebundenen 3. Arguments (hier: Attribuierung). Der semantisch-pragmatische Sinn dieses syntaktischen Verfahrens ist die Mit-Aktivierung der literalen Bedeutung, genauer gesagt: der übertragene Gebrauch der phraseologischen Bedeutung (s. Feilke 1996, S. 122). Das Ergebnis ist der kontextuell-situativ realisierte VT auf die indonesische Palme bringt - ein integrativ valenztheoretisches Produkt kreativ-wortspielerischer Motivation. $^{36 *}$

\section{Literatur}

Ágel, Vilmos (1997): Reflexiv-Passiv, das (im Deutschen) keines ist. Überlegungen zu Reflexivität, Medialität, Passiv und Subjekt. In: Dürscheid, Christa/Ramers, Karl Heinz/Schwarz, Monika (Hgg.): Sprache im Fokus. Festschrift für Heinz Vater zum 65. Geburtstag. Tübingen. S. 147-187.

Ágel, Vilmos (2000): Valenztheorie. Tübingen. (Narr Studienbücher).

Ágel, Vilmos (2003): Wort- und Ausdrucksvalenz(träger). In: Cornell, Alan/Fischer, Klaus/Roe, F. Ian (eds.): Valency in Practice/Valenz in der Praxis. Oxford usw. (German Linguistic and Cultural Studies 10), S. 17-36.

Ágel, Vilmos (2003a): Polylexikalität oder am Anfang waren mindestens zwei Wörter. Über eine Grundfrage (nicht nur) der Phraseologie. Budapest. [erscheint in der Festschrift für Regina Hessky].

Blume, Kerstin (2000): Markierte Valenzen im Sprachvergleich: Lizenzierungs- und Linkingbedingungen. Tübingen. (Linguistische Arbeiten 411).

Burger, Harald (1989): „Bildhaft, übertragen, metaphorisch ...“. Zur Konfusion um die semantischen Merkmale von Phraseologismen. In: Gréciano, Gertrud (Hg.): EUROPHRAS 88. Phraséologie Contrastive. Actes du Colloque International Klingenthal - Strasbourg 12-16 mai 1988. Strasbourg. (Collection Recherches Germaniques 2), S. 17-29.

Burger, Harald (1998). Phraseologie. Eine Einführung am Beispiel des Deutschen. Berlin. (Grundlagen der Germanistik 36).

${ }^{36}$ Der Fall auf die Palme bringen steht für Fix'sche Konstruktionsmodelle. Im Falle von Konstruktionsgerüsten wie z. B. jmdn. außer Gefecht setzen sehe ich zwei theoretische Möglichkeiten: (1) Wenn kein Valenzrealisierungsmuster mit $\mathrm{PO}_{\text {auBer }}$ angenommen wird, kann das 3 . Argument weder geöffnet noch geschlossen oder freigesetzt werden. Es bleibt als syntaktisches Unikat außerhalb der Abgleichung; (2) Wenn kein 3. Argument außer Gefecht in der Binnenstruktur des PH angenommen wird, bleibt die PP außer Gefecht als lexikalisches Unikat außerhalb der Abgleichung.

* Ich danke für die Unterstützung, die mir im Rahmen eines SZPÖ bzw. von OTKA (T 034340 NYE) zuteil wird. Für wichtige Hinweise danke ich Klaus Fischer und Jürgen Erich Schmidt, für Hilfe bei der Erstellung von Tabellen und Darstellungen, die allerdings in der Druckfassung teilweise ausgespart bleiben mussten, Annamária Fótos und Katalin Szécsi. 
Coseriu, Eugenio (1967): Lexikalische Solidaritäten. In: Poetica 1, S. 293-303.

Coulmas, Florian (1985): Diskursive Routine im Fremdsprachenerwerb. In: Sprache und Literatur 56, S. 47-66.

Dobrovol'skij, Dmitrij O. (1995): Kognitive Aspekte der Idiom-Semantik. Studien zum Thesaurus deutscher Idiome. Tübingen. (Eurogermanistik 8).

Dobrovol'skij, Dmitrij O. (2000): Gibt es Regeln für die Passivierung deutscher Idiome? In: Das Wort. Germanistisches Jahrbuch GUS 1999, S. 21-40.

Eisenberg, Peter (1994): Grundriß der deutschen Grammatik. 3., überarb. Aufl. Stuttgart/ Weimar.

Engel, Ulrich (1994): Syntax der deutschen Gegenwartssprache. 3., völlig neu bearb. Aufl. Berlin. (Grundlagen der Germanistik 22).

Feilke, Helmuth (1996). Sprache als soziale Gestalt. Ausdruck, Prägung und die Ordnung der sprachlichen Typik. Frankfurt am Main.

Fischer, Klaus (2001): Noch immer: Ergänzungen und Angaben. In: Sprachwissenschaft 26, S. 239-268.

Fischer, Klaus (2003): Verb, Aussage, Valenzdefinition und Valenzrealisierung: auf dem Weg zu einer typologisch adäquaten Valenztheorie. In: Coene, Ann/Van Pottelberge, Jeroen/Willems, Klaas (Hgg.): Valenztheorie: Neuere Perspektiven. Gent. (Themenheft der Studia Germanica Gandensia). [erscheint].

Fix, Ulla (1974/76): Zum Verhältnis von Syntax und Semantik im Wortgruppenlexem. In: Beiträge zur Geschichte der deutsche Sprache und Literatur 95, S. 214-318 und 97, S. 7-78.

Fix, Ulla (1979): Zum Verhältnis von Syntax und Semantik im Wortgruppenlexem. In LS/ ZISW/A 56, S. 1-19.

Fleischer, Wolfgang (1997): Phraseologie der deutschen Gegenwartssprache. 2., durchgesehene und ergänzte Aufl. Tübingen.

Gansel, Christina (2003): Valenz und Kognition. In: Ágel, Vilmos/Eichinger, Ludwig M./ Eroms, Hans Werner/Hellwig, Peter/Heringer, Hans Jürgen/Lobin, Henning (Hgg.): Dependenz und Valenz. Ein internationales Handbuch der zeitgenössischen Forschung. Bd.1. Berlin/New York. (Handbücher zur Sprach- und Kommunikationswissenschaft). 1-43. [im Druck, Paginierung gilt nur für das Manuskript].

Gréciano, Gertrud (1992): Zum System der Phrasemverwendung. In: Földes, Csaba (Hg.): Deutsche Phraseologie in Sprachsystem und Sprachverwendung. Wien. S. 149-169.

Heringer, Hans Jürgen (1984): Neues von der Verbszene. In: Stickel, Gerhard (Hg.): Pragmatik in der Grammatik. Jahrbuch 1983 des Instituts für deutsche Sprache. Düsseldorf. (Sprache der Gegenwart 60). S. 34-64.

Heringer, Hans Jürgen (1985): The Verb and its Semantic Power: Association as a Basis for Valency Theory. In: Journal of Semantics 4, S. 79-99.

Hessky, Regina (1988): Verbale Phraseologismen: valenzkonform oder nicht? In: Mrazović, Pavica/Teubert, Wolfgang (Hgg.): Valenzen im Kontrast. Ulrich Engel zum 60. Geburtstag. Heidelberg. S. 139-149.

Hyvärinen, Irma (2003): Der verbale Valenzträger. In: Ágel, Vilmos/Eichinger, Ludwig M./Eroms, Hans Werner/Hellwig, Peter/Heringer, Hans Jürgen/Lobin, Henning (Hgg.): Dependenz und Valenz. Ein internationales Handbuch der zeitgenössischen Forschung. Bd.1. Berlin/New York. (Handbücher zur Sprach- und Kommunikationswissenschaft). S. 1-93. [im Druck, Paginierung gilt nur für das Manuskript].

Iker, Bertalan (1996): Zur Passivbildung verbaler Idiome. In: Jahrbuch der ungarischen Germanistik 1996, S. 223-236.

Jacobs, Joachim (1994): Kontra Valenz. Trier. (Fokus 12).

Jacobs, Joachim (1994a): Das lexikalische Fundament der Unterscheidung von obligato- 
rischen und fakultativen Ergänzungen. In: Zeitschrift für germanistische Linguistik 22, S. 284-319.

Keil, Martina (1997): Wort für Wort. Repräsentation und Verarbeitung verbaler Phraseologismen (Phraseo-Lex). Tübingen. (Sprache und Information 35).

Keller, Rudi (1990): Sprachwandel. Von der unsichtbaren Hand in der Sprache. Tübingen. (UTB 1567).

Kolde, Gottfried (1979): Zur Valenz fester verbaler Syntagmen. In: Löffler, Heinrich/Pestalozzi, Karl/Stern, Martin (Hgg.): Standard und Dialekt. Studien zur gesprochenen und geschriebenen Gegenwartssprache. Festschrift für Heinz Rupp zum 60. Geburtstag. Bern/München. S. 73-87.

Korhonen, Jarmo (1988): Valenz und Verbidiomatik. In: Helbig, Gerhard (Hg.): Valenz, semantische Kasus und/oder "Szenen“. Berlin. (Linguistische Studien A 180). S. $105-118$.

Korhonen, Jarmo (1995): Morphosyntaktische Variabilität von Verbidiomen. In: Korhonen, Jarmo: Studien zur Phraseologie des Deutschen und des Finnischen. Bochum (Studien zur Phraseologie und Parömiologie 7). S. 67-93. [Erstveröffentlichung 1992].

László, Sarolta (1988): Mikroebene. In: Mrazović, Pavica/Teubert, Wolfgang (Hgg.): Valenzen im Kontrast. Ulrich Engel zum 60. Geburtstag. Heidelberg. S. 218-233.

Moon, Rosamund (1998): Frequencies and Forms of Phrasal Lexemes in English. In: Cowie, Anthony Paul (ed.): Phraseology: theory, analysis, and applications. Oxford (Oxford studies in lexicography and lexicology). S. 79-100.

Sadziński, Roman (1989): Statische und dynamische Valenz. Probleme einer kontrastiven Valenzgrammatik Deutsch-Polnisch. Hamburg. (Beiträge zur Sprachwissenschaft 1).

Schemann, Hans (1987): Was heißt ,Fixiertheit " von phraseologischen oder idiomatischen Ausdrücken? In: Korhonen, Jarmo (Hg.): Beiträge zur allgemeinen und germanistischen Phraseologieforschung. Internationales Symposium in Oulu 13.-15. Juni 1986. Oulu. (Veröffentlichungen des Germanistischen Institus 7). S. 23-36.

Stein, Stephan (1995): Formelhafte Sprache. Untersuchungen zu ihren pragmatischen und kognitiven Funktionen im gegenwärtigen Deutsch. Frankfurt am Main usw. (Sprache in der Gesellschaft. Beiträge zur Sprachwissenschaft 22).

Torzova, M. V. (1983): Zur Valenz der Phraseologismen. In: DaF 20, S. 283-287.

Van Pottelberge, Jeroen (2001): Verbonominale Konstruktionen, Funktionsverbgefüge. Vom Sinn und Unsinn eines Untersuchungsgegenstandes. Heidelberg. (Germanistische Bibliothek 12).

Welke, Klaus (1994): Valenz und Satzmodelle. In: Thielemann, Werner/Welke, Klaus (Hgg.): Valenztheorie - Werden und Wirkung. Wilhelm Bondzio zum 65. Geburtstag. Münster. S. 227-244.

Welke, Klaus (2002): Deutsche Syntax funktional. Perspektiviertheit syntaktischer Strukturen. Tübingen. (Stauffenburg Linguistik 22).

Wotjak, Barbara (1992): Verbale Phraseolexeme in System und Text. Tübingen. (RGL 125). Zifonun/IdS-Grammatik (1997) = Zifonun, Gisela (1997): E2 2. Komplemente. In: Zifonun, Gisela/Hoffmann, Ludger/Strecker, Bruno: Grammatik der deutschen Sprache. 3 Bde. Berlin/New York. (Schriften des Instituts für deutsche Sprache 7). S. 1027-1118. 